\title{
Optical Spectroscopy for the Quality Control of ATMP fabrication: A new method to monitor cell expansion and to detect contaminations
}

\author{
B. Wacogne ${ }^{1,2}$, D. Legrand ${ }^{1}$, C. Pieralli ${ }^{1}$, A. Frelet-Barrand ${ }^{1}$ \\ ${ }^{I}$ FEMTO-ST institute, Univ. Bourgogne Franche-Comte, CNRS, 15B avenue des Montboucons, 25030 Besancon, cedex, \\ France \\ ${ }^{2}$ Centre Hospitalier Universitaire de Besançon, Centre d'Investigation Clinique, INSERM CIC 1431, 25000, Besançon, \\ France \\ \{bruno.wacogne, christian.pieralli\}@univ-fcomte.fr, \{deborah.legrand, annie.frelet-barrand\}@femto-st.fr
}

Keywords: Optical spectroscopy, advanced therapy medicinal product, cell growth monitoring, contamination detection.

\begin{abstract}
Fabrication of Advanced Therapy Medicinal Products takes currently place in clean and sterile environment and is highly sensitive to any contamination sources. It lasts for several days and is extremely expensive. Quality controls must be performed throughout the process, especially to monitor cell growth during the expansion phase and to detect any contaminations. Regular sampling of the bioreactor's content is required and subsequent biological investigation are conducted. Major drawbacks are first, a delayed result of the quality control and second, an added risk to induce new contaminations when sampling the content of the bioreactor. Here, we present a real time optical spectroscopy method which can be used in a closed system manner, hence reducing the risk of sampling-related contamination. Analysing the shape of the absorption spectrum of the bioreactor content allows monitoring the cell growth and alerting users in case of contamination. Cell concentrations are measured with an accuracy of $\pm 5 \%$ and contamination can be detected about 3 hours after it occurred. This allows stopping the fabrication as soon as a problem arises leading to several tens of thousand dollars savings. Consequently, the price of these products should be greatly reduced and they may be proposed to more patients.
\end{abstract}

\section{INTRODUCTION}

ATMPs (Advanced Therapy Medicinal Products) have recently emerged to offer new treatment solutions for patients with no further therapeutic options. For some, they are based on the use of "drug" cells derived from genetic modification or tissue and cell engineering. These "living" drugs are subject to substantial manipulations that allow cells to acquire new physiological functions, biological characteristics or reconstruction properties. The development of new biological drugs is inspired by the natural processes of the body such as the use of stem cells for tissue regeneration, lymphocytes for cancer immunotherapy or apoptotic cells for antiinflammatory purposes.

However, the fabrication of these drugs requires the implementation of complex technologies of cell sorting, amplification, genetic transduction, amplification-division, activation, and this at several stages of production and in sterile clean room type environment. As for industrial products, their production is expensive, mainly because of the complex infrastructure required, the time needed to complete the production stages and the complex quality control processes. A schematic description of the fabrication process of CAR-T cells is given in figure 1 (Wang 2016). It also applied to other ATMPs fabrications.

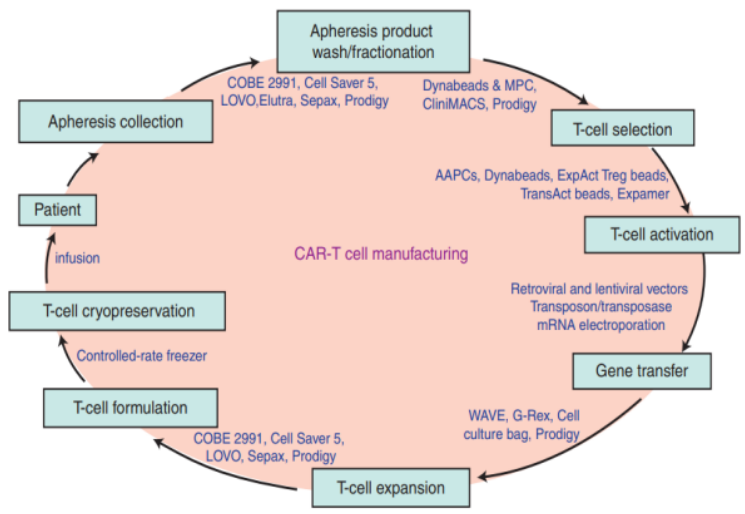

Figure 1: Fabrication process of CAR-T cells (Wang 2016). 
The basic principle consists in sampling the patient's blood and extract the cells of interest (T cells for figure 1). These cells are transduced to acquire the desired therapeutic properties. A sorting step then makes it possible to keep only the correctly transduced $\mathrm{T}$ cells. From this moment, these genetically modified cells are amplified/expanded in a bioreactor for a period that can extend up to 1 week. At the end of the process, the ATMP is injected to the patient or cryo-preserved before injection. The goal of our project (see acknowledgements) is to realize all these operations within a miniaturized and autonomous device. One of the main constraints that needs to be lifted is the following.

The requirement of no contamination of the products requires working in a controlled environment and preserving the closed system as often as possible. This is frequently made difficult by the absence of containers, reagents or materials adapted to the protocol. This constraint implies many samplings during the protocol such as sampling for the evaluation of bacteriological contamination. This increases the risk of contamination, the time of completion and requires increased traceability. These evaluations being long, the fabrication process continues in parallel, sometimes requiring to stop production after several days in case of a contamination. These stops actually increase the cost and delay or even stop the delivery of the drug to the patient.

The work presented in this paper addresses the constraint of closed system and real time control of what happens in the bioreactor during the expansion phase. The goal is twofold, monitor the cell growth and detect early contaminations. In-line or real time measurement techniques have been widely studied either for cell culture or contaminant development monitoring, very rarely for both.

For example concerning cell culture, various techniques have been presented in Teixeria review (Teixeria, 2009). Techniques based on ultrasonic measurements (Melchor, 2018) or capacitive techniques (Lee, 2016) have been proposed. Impedance monitoring, either in a bulk system (Cacopardo, 2019) or in a microfluidic chip (Fong Lei, 2014) has also been presented. In these references however, indications whether or not the proposed method can be adapted in a closed system configuration were not discussed.

Concerning bacteria detection or monitoring, different sensors to detect Escherichia coli (hereafter E. coli) have been proposed by Ikonen (Ikonen, 2017). These bacteria can also be detected using modified Field Effect Transistors (Thakur, 2018).
Detecting several contaminants with a single device has been demonstrated. The use of fiber optic Fourier Transform Infra-Red spectroscopy (Hassan, 2016), quartz crystal sensors (Chang, 2006), and electrochemistry (Safavieh, 2014) has been reported.

Only very few papers mention both cell monitoring and contaminant detection. For example in (Liu, 2017), advanced signal processing applied to Raman spectroscopy has been proposed. Together with normal operation condition monitoring, authors demonstrated the detection of growth problems 5 hours after they stopped feeding the cells. They also detected effects of contamination with their monitoring algorithm. However, the nature of the contamination and the time required to detect it was not specified.

In this paper, we propose a proof of concept based on white light absorption spectroscopy used to continuously monitor the evolution of cells concentration in a bioreactor and to issue an alarm signal about 3 hours after a contamination occurred. The next section of the paper describes the experimental set-up and biological samples used in this study. Section 3 presents the results obtained using two methods based on spectra shape analysis and Principal Component Analyses (PCA) respectively. Short discussions of these early results and aspects concerning socio-economic impacts will be given in section 4 .

\section{MATERIALS AND METHODS}

For this proof of concept, measurements are not performed in a closed system configuration. Adaptation of the method in this particular environment is shortly discussed in section 4 .

\subsection{Experimental set-up}

The extremely simple experimental set-up is schematically presented in figure 2 .

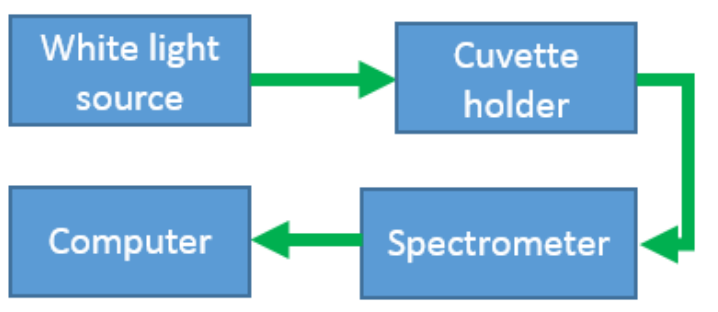

Figure 2: Description of the experimental set-up. 
The set-up was composed of a white light source (Ocean Optics HL 2000) connected to a cuvette holder (Avantes CUV-UV/VIS) via conventional step index optical fibers (Thorlabs M25L01). After propagation through the cuvette, light was launched into a spectrometer for absorption spectra acquisition (Ocean Optics QE-Pro). Fluorimeter polymethacrylate cuvettes were filled up to $3 \mathrm{~mL}$ with solutions of cells and bacteria (Sigma-Aldricht C0793-100EA).

Absorption spectra were measured using the specific feature available in the SpectraSuite software from Ocean Optics. Reference was obtained with a cuvette filled with PBS only. After transfer to PC, data processing was performed using Matlab ${ }^{\mathrm{TM}}$ $\mathrm{R} 2014 \mathrm{~b}$ version. Spectra used in section 3.2 were slightly smoothed using a cubic spline algorithm in order to maximize the $\mathrm{R}^{2}$ of the spectra fittings. Principal Component Analysis was performed with smoothed and normalized spectra.

\subsection{Lymphocyte and E. coli preparation}

Lymphocyte cell lines (Ramos, ATCC, USA) were cultured in X-Vivo (Lonza, Switzerland) with 5\% FBS $\quad\left(\right.$ Gibco $\left.^{\mathrm{TM}} \quad 10270106\right)$ and $10 \%$ streptomycine/penicillin $(100 \mu \mathrm{g} / \mathrm{mL}+100 \mathrm{UI} / \mathrm{mL}$, CABPES01-0U, Eurobio) in a humidified $37^{\circ} \mathrm{C}, 5 \%$ $\mathrm{CO}_{2}$ incubator. Cells were recovered after 2-3 days culture by centrifugation at $700 \mathrm{~g}, 10 \mathrm{~min}, 25^{\circ} \mathrm{C}$. Different cell concentrations $\left(10^{4} \times[1,2,4,6,8,10\right.$, 20, 30, 40, 50, 60, 70, 80, 90, 100] cells/mL) were prepared after dilution in autoclaved PBS 1x pH7.4 (Sigma, USA).

Escherichia coli DH5 $\alpha$ (NEB, USA) were cultured in Luria Bertani overnight at $37^{\circ} \mathrm{C}, 180 \mathrm{rpm}$ in a MaxQ incubator. They were recovered by centrifugation at $5000 \mathrm{~g}, 15 \mathrm{~min}, 20^{\circ} \mathrm{C}$ and resuspended in autoclaved PBS 1x pH7.4 (Sigma, USA). Optical density of the re-suspension was measured in a spectrophotometer Shimadzu at 595 nm. Afterwards, different bacteria concentrations $\left(10^{6} \times[1,2,4,6,8,10,20,30,40,50,60,70,80,90\right.$, $100]$ bacteria/mL) were prepared for experiments.

In this work, so-called contaminated spectra presented below are artificial and made by adding spectra of lymphocyte and E. coli. This aspect will be discussed in section 4 .

\section{EXPERIMENTAL RESULTS}

\subsection{Calculating concentrations of both species: a difficult way}

Examples of spectra recorded with lymphocytes and E. coli are given in figure 3 .

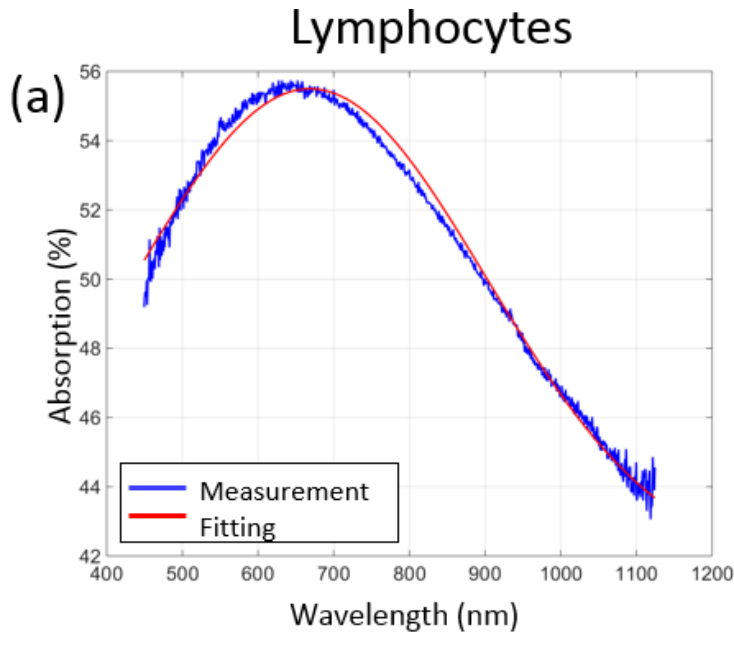

E. coli

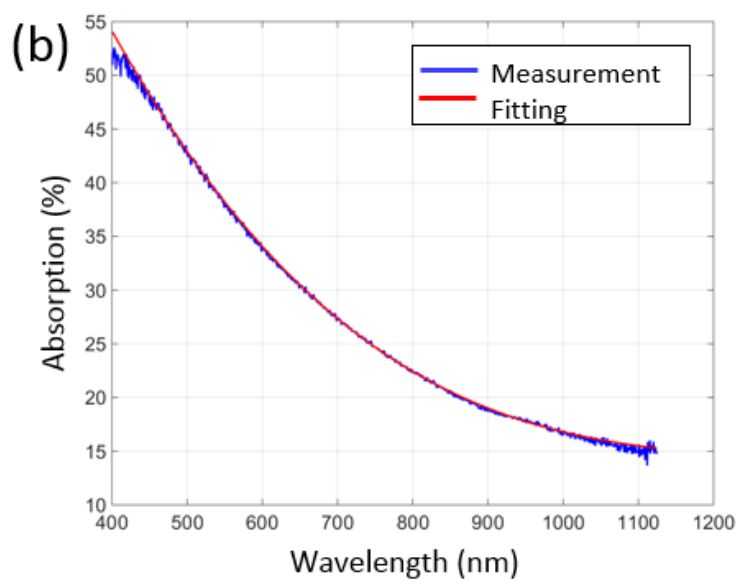

Figure 3: Examples of absorption spectra. (a) Lymphocytes, (b) E. coli.

Noting that the shapes of the spectral absorptions of the two species are different, the goal was to measure the absorption spectrum of the contents of the bioreactor continuously. For each recorded spectrum, the idea is to analyse the shape, to separate the part due to the contribution of lymphocytes from that due to $E$. coli and to calculate their respective concentrations.

To do this, it was necessary to determine the evolution of the spectra of the two species as a function of the concentration. Concentration ranges 
of the two species were used and, by fitting, it was possible to express their spectral evolutions as a function of their concentrations.

Equations (1) to (5) represents the evolution for lymphocytes.

$$
\operatorname{Spec}_{\text {lym }}(\lambda)=p 1 \cdot \exp \left(-\left(\frac{\lambda-\mathrm{p} 2}{\mathrm{p} 3}\right)^{2}\right)
$$

Here, $\lambda$ is the wavelength and functions "pi" functions are given by:

$$
\begin{gathered}
\text { p1 }=\text { a1. exp }\left(-\left(\frac{\mathbf{C}-\mathrm{a} 2}{\mathrm{a} 3}\right)^{2}\right) \\
\text { p2 }=\mathrm{b} 1 \cdot \mathbf{C}+\mathrm{b} 2 \\
\text { p3 }=\mathrm{c} 1 \cdot \mathbf{C}^{2}+\mathrm{c} 2 \cdot \mathbf{C}+\mathrm{c} 3 \\
\text { p4 }=\mathrm{d} 1 \cdot \mathbf{C}^{2}+\mathrm{d} 2 \cdot \mathbf{C}+\mathrm{d} 3
\end{gathered}
$$

where $\mathbf{C}$ is the lymphocytes concentration.

Equations (6) to (10) represents the evolution for lymphocytes.

$$
\operatorname{Spec}_{\text {E.Coli }}(\lambda)=q 1 \lambda^{3}+q 2 \lambda^{2}+q 3 \lambda+q 4
$$

Here, $\lambda$ is the wavelength and functions "qi" functions are given by:

$$
\begin{gathered}
\mathrm{q} 1=\mathrm{e} 1 \cdot \mathbf{C}^{2}+\mathrm{e} 2 \cdot \mathbf{C}+\mathrm{e} 3 \\
\mathrm{q} 2=\mathrm{f} 1 \cdot \mathbf{C}^{2}+\mathrm{f} 2 \cdot \mathbf{C}+\mathrm{f} 3 \\
\mathrm{q} 3=\mathrm{g} 1 \cdot \mathbf{C}^{2}+\mathrm{g} 2 \cdot \mathbf{C}+\mathrm{g} 3 \\
\mathrm{q} 4=\mathrm{h} 1 \cdot \mathbf{C}^{2}+\mathrm{h} 2 \cdot \mathbf{C}+\mathrm{h} 3
\end{gathered}
$$

\begin{tabular}{|c|c|c|c|}
\hline p1 & $\mathrm{a} 1=14.32$ & $\mathrm{a} 2=4.1 \times 10^{5}$ & $a 3=6.145 \times 10^{5}$ \\
\hline p2 & $\mathrm{b} 1=7.21 \times 10^{-5}$ & b2 $=647$ & \\
\hline p3 & $\mathrm{c} 1=1 \times 10^{-10}$ & $c 2=-1.9 \times 10^{-5}$ & $\mathrm{c} 3=325.7$ \\
\hline $\mathrm{p} 4$ & $\mathrm{~d} 1=-5 \times 10^{-11}$ & $\mathrm{~d} 2=1.3 \times 10^{-3}$ & $\mathrm{~d} 3=6.791$ \\
\hline $\mathrm{q} 1$ & $\mathrm{e} 1=1.2 \times 10^{-23}$ & $\mathrm{e} 2=-1.6 \times 10^{-15}$ & $\mathrm{e} 3=1.2 \times 10^{-9}$ \\
\hline $\mathrm{q} 2$ & $\mathrm{f} 1=-3.3 \times 10^{-20}$ & $\mathrm{f} 2=5.1 \times 10^{-12}$ & $\mathrm{f} 3=-1.7 \times 10^{-6}$ \\
\hline $\mathrm{q} 3$ & $\mathrm{~g} 1=2.8 \times 10^{-17}$ & $\mathrm{~g} 2=-5.5 \times 10^{-09}$ & $\mathrm{~g} 3=-8.3 \times 10^{-4}$ \\
\hline $\mathrm{q} 4$ & $\mathrm{~h} 1=-1 \times 10^{-14}$ & $\mathrm{~h} 2=2.4 \times 10^{-6}$ & $\mathrm{~h} 3=1.234$ \\
\hline
\end{tabular}

where $\mathbf{C}$ is the $E$. Coli concentration.

Coefficients used in these equations are given in table 1 .

Table 1: Coefficients used in the above equations.

These evolutions are represented in figure 4 (they were used to fit the experimental spectra presented in figure 3 (red curves)).

From there, for each recorded spectrum and considering either lymphocytes or E. coli, we compared the actual concentration values with those calculated with the above functions by fitting the spectra. Figure 5 shows the results obtained.

It was found that the exploitation of the shape of the spectra to calculate concentrations was effective only for lymphocyte concentrations ranging from $10^{5}$ to $9 \times 10^{5}$ cell $/ \mathrm{mL}$ (black circles in figure $5(\mathrm{a})$ where the fitting was not effective). In the validity range, an accuracy of $\pm 5 \%$ was measured.

\section{Lymphocytes}

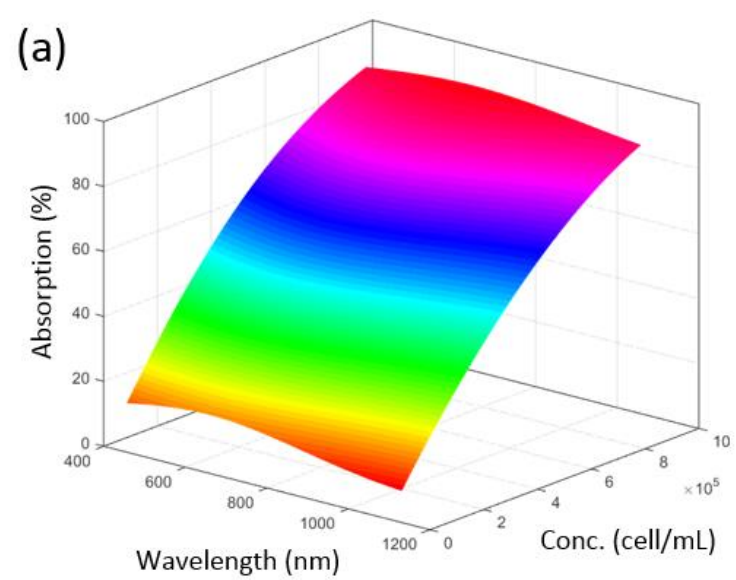

\section{E. coli}

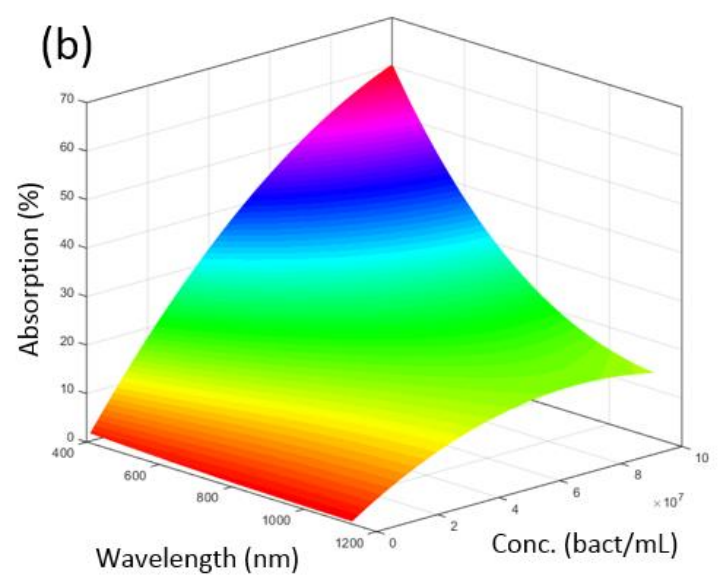

Figure 4: Theoretical evolutions of the absorption spectra with concentrations. (a) Lymphocytes, (b) E. coli.

We have also studied other descriptors of the spectra. By considering their associated colour, it is shown that, in the HSV base, the "Value" variable made it possible to describe the concentrations of the two species. The same is true if we consider the entropy of spectra. However, when considering spectra of lymphocytes and E. coli mixtures, it is extremely difficult to separate their respective contributions. The problem becomes insoluble when several types of pathogens are considered.

Indeed, as long as only lymphocytes are present in the bioreactor (no contamination), fitting the shape of the spectra to monitor the cell growth leads to the same accuracy as what we obtained using 
conventional turbidimetry as presented in figure 6 . For the concentration range considered, the area under the spectra can be modelled using a second order polynomial function.
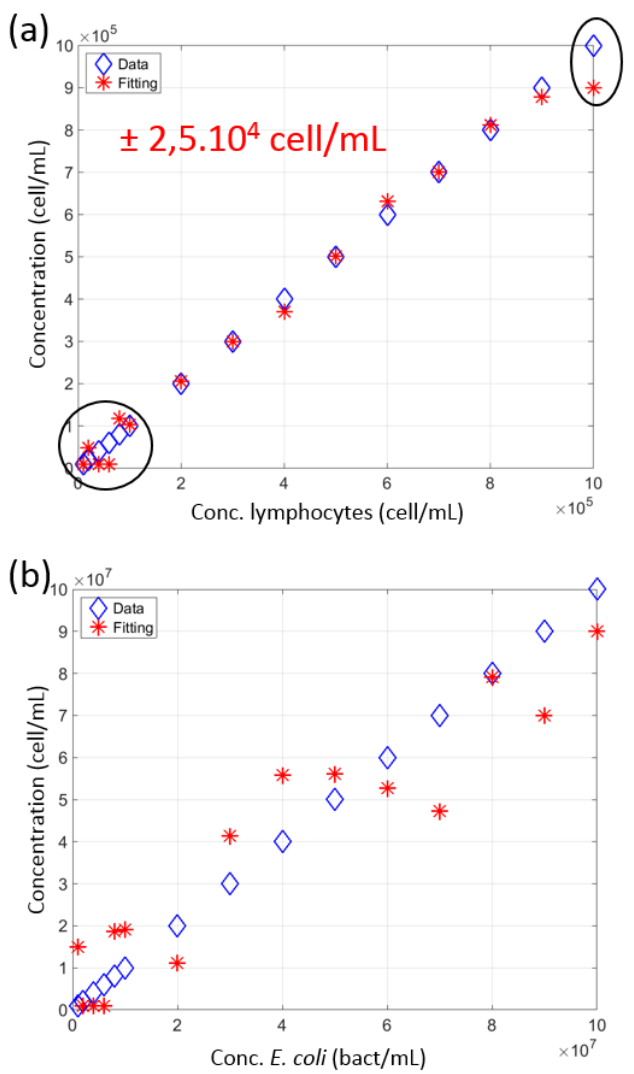

Figure 5: Calculating the concentration from the shape of experimental spectra (a) Lymphocytes, (b) E. coli. Blue diamonds: real concentrations, red crosses: fitted concentrations. Black circles in (a): concentration ranges for which the method does not work.

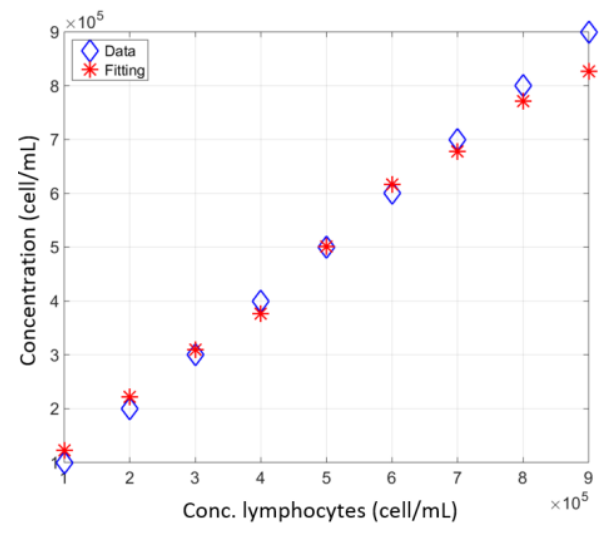

Figure 6: Calculating the lymphocyte concentrations from the area under the spectra (turbidimetry). Blue diamonds: real concentrations, red crosses: fitted concentrations.
However, detecting contaminations can be achieved by considering things differently. This is the subject of the next section.

\subsection{Monitoring cell concentrations and detecting contaminations: a new approach}

\subsubsection{Fitting the shape of the absorption spectra}

We must understand the problem differently. During the expansion phase, as long as everything is normal (no contamination), cells concentration can be monitored using turbidimetry. Now, if contamination occurs, the shape of the spectrum resulting from the contribution of the lymphocytes and the contaminant differs from the ones corresponding to lymphocytes alone.

The idea is this. During the expansion phase, absorption spectra are recorded and fitted with a function representing the shapes of the lymphocytes spectra when they are alone. An accurate fitting (high $\mathrm{R}^{2}$ ) means that no contamination has occurred and lymphocyte concentration is calculated by integrating the area under the spectra. On the contrary, a bad fitting ( $\mathrm{R}^{2}$ less than a threshold to be determined) means that contamination occurred indicating that the production must be stopped.

This can be achieved using a much simpler equation than the one depicted in figure 4(a). Indeed, we do not calculate a concentrations, we only fit the shape of the recorded spectrum. The following spectrum description can efficiently be used.

$$
A b s(\lambda)=a \cdot \exp \left(-\left(\frac{\lambda-b}{c}\right)^{2}\right)+d
$$

The goal is to fit any spectra with this equation. To do this, constraints were put to coefficients $a, b, c$ and d. Otherwise, the fitting algorithm ("trust region" in Matlab ${ }^{\text {TM }}$ Curfitting toolbox) always find set of parameters to describe even contaminated spectra. The fitting bounds and starting points are summarized in table 2 . 
Table 2: Bounds and starting points for fitting lymphocytes spectra.

\begin{tabular}{|c|c|c|c|}
\hline Coefficient & $\begin{array}{c}\text { Lower } \\
\text { bound }\end{array}$ & $\begin{array}{c}\text { Upper } \\
\text { bound }\end{array}$ & $\begin{array}{c}\text { Starting } \\
\text { point }\end{array}$ \\
\hline a & 0 & 200 & 100 \\
\hline b & 600 & 750 & 675 \\
\hline c & 0 & 500 & 250 \\
\hline d & 0 & 200 & 100 \\
\hline
\end{tabular}

Figure 7 shows example of lymphocytes absorption spectra fittings using equation (11). Spectra were slightly smoothed as mentioned above.
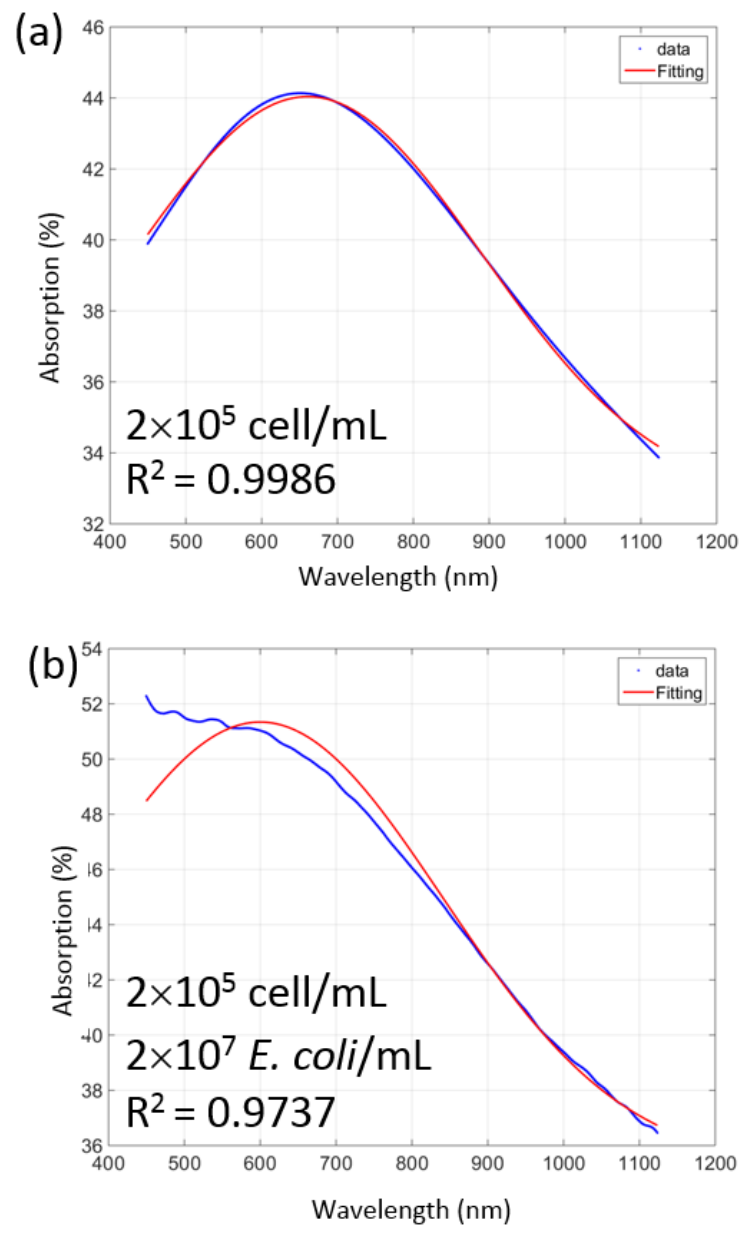

Figure 7: Examples of spectra fitting using equation (11). (a) Lymphocyte concentration $=2 \times 10^{5}$ cell $/ \mathrm{mL}, \mathrm{R}^{2}=$ 0.9986. (b) Lymphocyte concentration $=2 \times 10^{5}$ cell $/ \mathrm{mL}, E$. coli concentration $=2 \times 10^{7} \mathrm{bact} / \mathrm{mL}, \mathrm{R}^{2}=0.9737$.

Figure 7(a) shows a fitting performed with pure lymphocytes at $2 \times 10^{5}$ cell $/ \mathrm{mL}$ with $\mathrm{R}^{2}=0.9986$. Considering only pure lymphocyte solutions, $\mathrm{R}^{2}$ coefficients were always greater than 0.99 for except for $10^{4}$ and $10^{6}$ cell $/ \mathrm{mL}$. Indeed, for these concentrations, absorption is either too weak or too strong for our method to work. We did not consider these concentrations in what follows.

Figure 7(b) shows a fitting performed with the same lymphocyte concentration contaminated with $2 \times 10^{7} \mathrm{E}$. coli $/ \mathrm{mL}$. Because of the contamination, $\mathrm{R}^{2}$ decreases to 0.9737 .

The evolution of $\mathrm{R}^{2}$ with increasing concentrations of $E$. coli for different initial lymphocyte concentrations is shown in figure 8. Each curve corresponds to one lymphocyte concentration. For each lymphocytes concentration, the evolution of $\mathrm{R}^{2}$ is plotted as a function of $E$. coli concentration.

It was found that, whatever the lymphocyte concentration is, the bacterial detection limit was about $2.5 .10^{7}$ cells $/ \mathrm{mL}$ with a positivity threshold $\mathrm{R}^{2}$ $=0.988$ (arrows in the figure). Knowing that $E$. coli divides every $20 \mathrm{~min}$ and considering that the contamination is due to 1000 bacteria, the warning signal can be issued $4 \mathrm{~h} 52$ min post contamination.

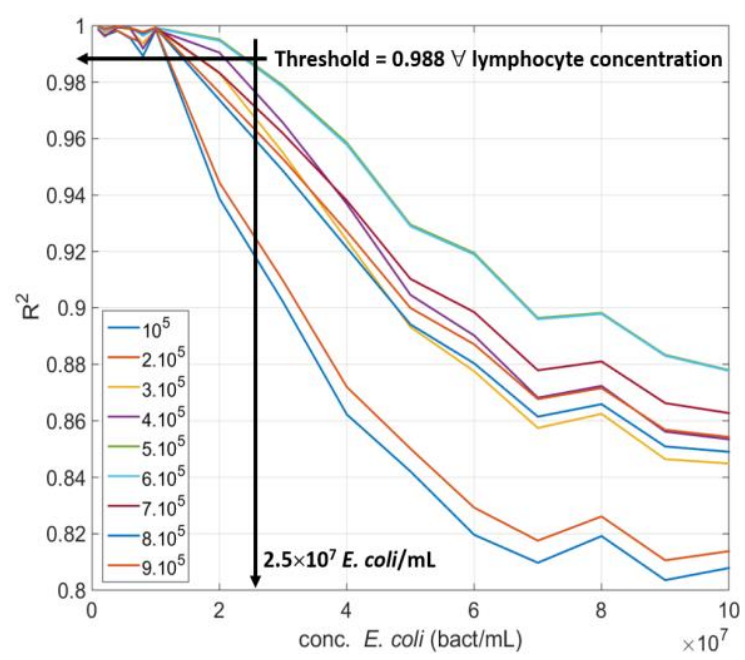

Figure 8: Evolution of the $\mathrm{R}^{2}$ coefficient with the concentration in E. coli for different lymphocytes concentrations. The legend corresponds to lymphocyte concentrations in cell $/ \mathrm{mL}$.

\subsubsection{Using Principal Component Analysis}

Principle Component Analysis was used to further reduce the time required to issue the alert signal. Spectra used here were smoothed and normalized as mentioned above.

First, it was applied to spectra of pure lymphocytes and E. coli. The result considering PC1 and PC2 is shown in figure 9. The two populations 
were clearly separated. The black line represents the frontier between these populations. It was defined by either the minima of the coordinates of lymphocytes (circles) and maxima of E. coli (crosses) or the analysis of the mean and variance of the two distributions. Taking into account these data, a straight line which separates the CP1-CP2 domain in two zones was defined (Janné, 2001).

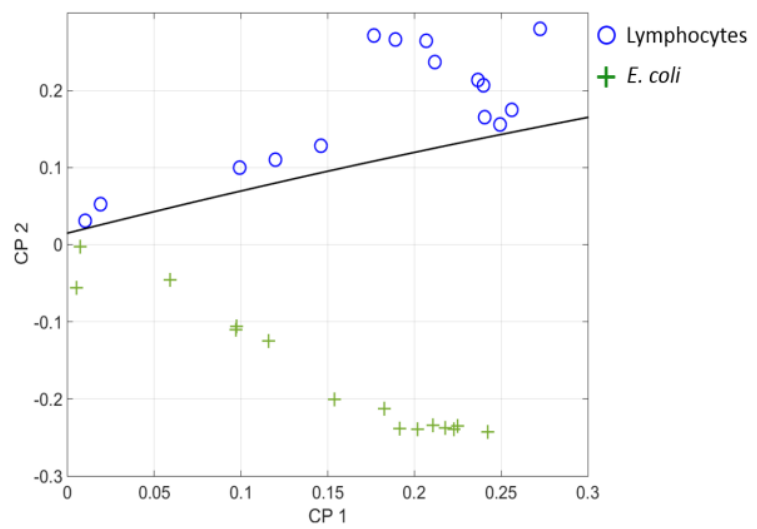

Figure 9: Principal Component Analysis. Blue circles: lymphocytes, green crosses: E. coli, black line: separation between lymphocytes and $E$. coli.

Second, we added the so-called contaminated spectra. The 30 data used to generate figure 9 form a base for the pure lymphocytes and E. coli populations. Contaminated spectra correspond to all possible combinations of lymphocyte spectra added to $E$. coli spectra. They were processed one by one. Each contaminated spectrum was considered as a $31^{\text {rst }}$ data in the above mentioned base before a new PCA was performed. This was iterated for the 225 possible combinations. The result is shown in figure 10 with the contaminated spectra marked with red squares.

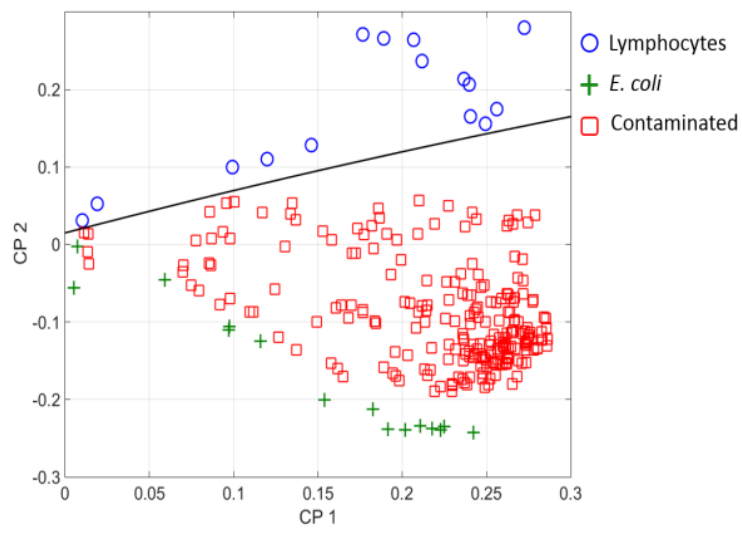

Figure 10: Principal Component Analysis. Blue circles: lymphocytes, green crosses: E. coli, red squares: contaminated cultures, black line: separation between pure and contaminated cultures.
Spectra corresponding to contaminated culture were all situated in the $E$. coli region. This means that the bacteria detection limit was $10^{6}$ cells $/ \mathrm{mL}$ (the minimum E. coli concentration considered in this study). Comparing with the method based on the spectra shape analysis and considering that the contamination is due to 1000 bacteria, the warning signal can now be issued about $3 \mathrm{~h} 19 \mathrm{~min}$ post contamination.

\section{DISCUSSION}

\subsection{Technical aspects}

Figures 5, 6, 8 and 10 show that analysing the absorption spectra of cells in culture not only allows monitoring the expansion phase during the fabrication of ATMPs, but also provides powerful tools to issue an alert signal about 3 hours after a contamination with 1000 bacteria occurred. These results were obtained using absorption spectra of various concentrations of lymphocytes and E. coli.

Bacterial contamination spectra are artificial spectra made by adding spectra of lymphocytes and E. coli. This could introduce a bias in the results presented here. Indeed, adding absorption spectra may lead to an artificial absorption greater than $100 \%$. However, the method based on the estimation of the shape of the absorption spectra will still be valid because only the shape is considered and not the value of the maximum absorptions. We recall that the $\mathrm{R}^{2}$ coefficient is only used to issue an alert signal. As long as no contamination is detected, equation depicted in figure 4 remains valid to monitor the expansion phase. This cell growth monitoring can also be performed considering conventional turbidimetry as mentioned above.

Results obtained using Principal Component Analysis do not suffer from this because it is performed using normalized spectra. In all cases, a more realistic study will involve real spectra recorded with actual mixture of lymphocytes and $E$. coli.

In this work, we only considered a contamination due to $E$. coli. We still need to extend this study to the case of other bacteria and other containments like yeasts and fungi. The methods presented here will still be valid as long as the shapes of the absorption spectra of contaminants are different enough from the ones of lymphocytes.

Also, the use of white light spectroscopy through conventional cuvettes makes possible an easy adaptation in a closed system configuration using a derivation from the bioreactor as proposed in figure 11(a). Note that it is also possible to envision the 
fabrication of a sterile absorption probe directly included in the bioreactor as depicted in figure 11(b).

(a)

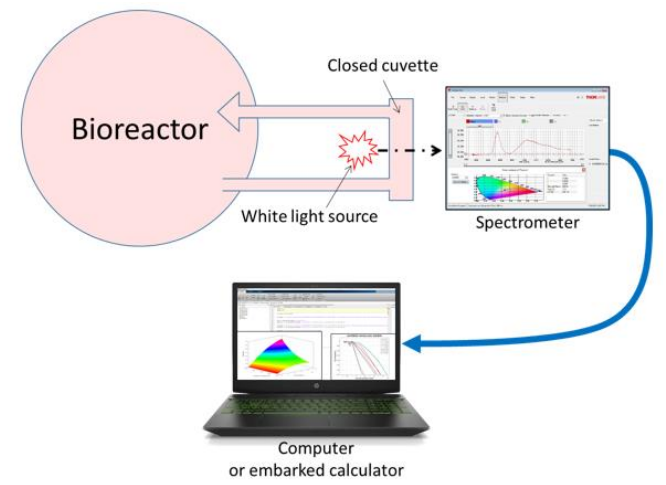

(b)

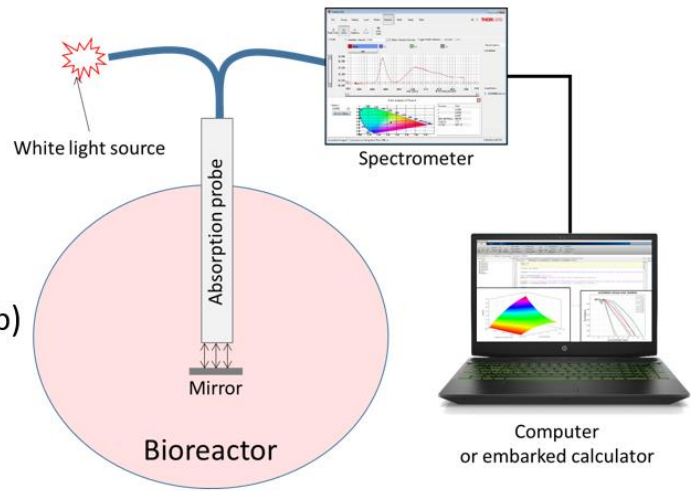

Figure 11: A possible integration of the method in a closed system environment. (a) Using a derivation. (b) Using a sterilized reflection probe coupled to a mirror.

\subsection{Socio-economic impacts}

As mentioned above, we shortly discuss socioeconomic aspects of this work. We recall that the duration of the expansion phase is several days. Each day increases the price of ATMPs and the quality control imposes regular samples, themselves risk of contamination. The results presented above are therefore particularly interesting since they allow quality control without sampling and make it possible to stop the expansion phase rapidly (and therefore the cost associated with inefficient manufacture) about than 3 hours post contamination if the latter is due to 1000 E. coli.

Designed to treat patients with pathologies that are currently incurable, ATMPs are likely to create a real therapeutic revolution in the coming years. It is currently difficult to estimate the number of pathologies that these medicines from the living can address and the number of patients likely to benefit.

At the industrial level, only a few ATMPs are available on the market and some have had their marketing authorization cancelled (Glybera,
Sipuleucel-T, ChondoCelect) mainly because of the enormous cost of their production. As far as we know, only the following treatments are available (note the price for a single treatment):

- $\quad$ KYMRIAH $(\$ 475,000)$

- YESCARTA $(\$ 373,000)$

- Strimvelis $(\$ 594,000)$

Included in these costs are losses due to fabrications, which are found to be contaminated during the final conformity test, and those due to latestage fabrication stops due to the scheduling of controls at precise dates.

Having a real-time and closed system monitoring and quality control method is of great interest in terms of research, industrial manufacturing and more importantly in terms of benefit to the patients.

\section{CONCLUSION}

In this paper, we have presented methods to continuously perform a quality control during the expansion phase of the fabrication of ATMPs and this in a closed system environment. These methods are based on analysing the absorption spectra of what happens in the bioreactor. Spectral shape analysis is used to monitor the cells growth and to issue an alert signal about $4 \mathrm{~h} 52$ post contamination. Principal Component Analysis does not allow determining the lymphocyte concentration but it allows reducing the time required to issue the alert signal to $3 \mathrm{~h} 19$ (durations calculated considering that contamination is due to $1000 \mathrm{E}$. coli). Advantages of such methods can be summarized as follows.

It is no longer necessary to sample the content of the bioreactor for analysis and detection of potential pathogens. There is no longer risk of product contamination due to sampling. The idea is no longer to seek to identify pathogens but just reveal the fact that the cell culture is not going as planned and stop production. The use of planned sampling at a fixed date and time without even knowing whether contamination will be detected is avoided. The cell growth is monitored in real time as long as the culture is normal. Contamination is detected extremely early. The production cost can be greatly reduced by stopping the production as soon as a contamination is detected

Indeed, in order to guarantee access to the largest number of patients, a new conception of the current mode of production and qualification of the ATMPs is necessary. Currently, our studies are focussed on 
the validation of these above described methods considering other types of pathogens.

\section{ACKNOWLEDGEMENTS}

This work was supported by the MiMedi project funded by BPI France (grant No. DOS0060162/00) and the European Union through the European Regional Development Fund of the Region Bourgogne-Franche-Comte (grant No. FC0013440).

\section{REFERENCES}

Cacopardo, L., et al, 2019. Real-time cellular impedance monitoring and imaging of biological barriers in a dualflow membrane bioreactor, Biosensors and Bioelectronics Vol. 140, pp. 111340

Chang, K-L., et al, 2006, Series quartz crystal sensor for remote bacteria population monitoring in raw milk via the Internet, Biosensors and Bioelectronics Vol. 21, pp. $1581-1590$

Fong Lei, K., et al, 2014. Real-time and non-invasive impedimetric monitoring of cell proliferation and chemosensitivity in a perfusion 3D cell culture microfluidic chip, Biosensors and Bioelectronics, Vol.51, pp.16-21

Hassan, M., et al, 2016. Detecting bacteria contamination on medical device surfaces using anintegrated fiberoptic mid-infrared spectroscopy sensing method, Sensors and Actuators B Vol. 231, pp. 646-654

Ikonen, J., et al, 2017. On-line detection of Escherichia coli intrusion in a pilot-scale drinking water distribution system », J. of Environmental Management, Vol. 198, pp. 384-392

Janné K. et al, 2001. Hierarchical principal component analysis (PCA) and projectionto latent structure (PLS) technique on spectroscopic data as adata pretreatment for calibration, J. Chemometrics, Vol. 15, pp. 203-213

Lee, S-L., et al, 2016. Real-time monitoring of 3D cell culture using a 3D capacitance biosensor, Biosensors and Bioelectronics, Vol. 77, pp. 56-61

Liu, Y-J, et al, 2017. Multivariate statistical process control (MSPC) using Raman spectroscopy for in-line culture cell monitoring considering time-varying batches synchronized with correlation optimized warping (COW), Analytica Chimica Acta Vol. 952, pp. 9-17

Melchor, J., et al, 2018. In-bioreactor ultrasonic monitoring of 3D culture human engineered cartilage, Sensors and Actuators B Vol. 266, pp. 841-852

Safavieh, M., et al, 2014. High-throughput real-time electrochemical monitoring of LAMP for pathogenic bacteria detection, Biosensors and Bioelectronics, Vol. 58, pp. 101-106

Teixeira, A.P., et al, 2009. Advances in on-line monitoring and control of mammalian cell cultures: Supporting the
PAT initiative, Biotechnology Advances, Vol. 27, pp. 726-732

Thakur, B. et al, 2018. Rapid detection of single E. coli bacteria using a graphene-based field-effect transistor device, Biosensors and Bioelectronics, Vol. 110, pp. $16-22$

Wang, X., et al, 2016. Clinical manufacturing of CAR T cells: foundation of a promising therapy, Molecular Therapy-Oncolytics, Vol. 3, pp. 16015 